\title{
Technical applications of an imaging Gamma-ray Compton Backscattering device and simulation using GEANT4
}

\section{Flechas*}

Departamento de Física, Universidad Nacional de Colombia, Bogotá D.C. 111321, Colombia

E-mail: DCFlechasG@unal . edu.co

\section{L.G. Sarmiento}

Departamento de Física, Universidad Nacional de Colombia, Bogotá D.C. 111321, Colombia

E-mail: LGSarmientoP@unal.edu.co, Luis.Sarmiento@nuclear.lu.se

\section{F. Cristancho}

Departamento de Física, Universidad Nacional de Colombia, Bogotá D.C. 111321, Colombia

E-mail: LFCristachomeunal.edu.co

\section{E. Fajardo}

Grupo de Física Nuclear de la Universidad Nacional de Colombia, Bogotá D.C. 111321,

Colombia

E-mail: Eduardo.Fajardo.Lopez@gmail.com

\begin{abstract}
The $\gamma$-backscattering imaging techniques are alternative methods to the transmission techniques to determine the amount and distribution of matter in objects. Images obtained with a $\gamma$ backscattering device are presented. In order to increase the understanding of the image formation process and to assist in the data analysis, a simulation of the camera was developed using the Geant4 simulation toolkit. Simulation and experimental results suggest that the device has promising potential industrial applications.
\end{abstract}

X Latin American Symposium on Nuclear Physics and Applications (X LASNPA),

1-6 December 2013

Montevideo, Uruguay

\footnotetext{
* Speaker.

${ }^{\dagger}$ Present address: Lund University, S-22100 Lund, Sweden.
} 


\section{Introduction}

When a collimated monoenergetic gamma-ray beam of intensity $I_{0}$ and energy $E$ traverses a distance $z$ of a given material, the transmitted intensity is $I_{T}(z)=I_{0} \exp (-\mu z)$ with $\mu$ the total lineal absorption coefficient of the material for the incident energy $E$. The simplicity of the previous relationship allows powerful applications in a very diverse range of fields, from the determination of thicknesses in industrial processes to obtaining images in medical diagnostics as well as in the petroleum industry. The geometrical characteristic of the transmission experiments is that the sample lies between the source and the detector. Although the number of application cases for transmission is quite large, there are however instances in which this arrangement is not possible. In very important cases the only chance is to place detector and source at the same side of the sample, which defines the Gamma-ray Compton Backscattering (GCB) technique. The number of backscattered photons cannot be calculated in such a simple way as in the transmission case because the photons that arrive to the detector may have undergone one (Single Scattering $=$ SS) or many Compton scattering processes (Multiple Scattering = MS) and an exact analytical computation is practically impossible because of the complex random nature of the whole process. Existing numerical algorithms (example: Collapsed Cone Convolution [1]), based on analytical descriptions, able to reproduce the energy and space distributions in clinical applications involve highly complex and time-consuming computations and seem too far-reaching for our present purposes. The described facts impose a more phenomenological path in order to investigate the properties of the backscattered intensity. The present work, a continuation of Ref. [2], is based on the use of a GCB imaging device proposed in Ref. [3]. The next section explains briefly its physical basis. The following sections give an account of several experimental determinations compared with state-of-the-art Monte Carlo simulations.

\section{The work principle of the Compton Camera}

The physical principle of the Compton Camera and the process to construct an image in a typical situation in which the camera can be useful -locating concealed objects- is shown in Figure 1. Positron annihilation from a ${ }^{22} \mathrm{Na}$ source produces two nearly collinear back-to-back $511 \mathrm{keV}$ photons. $\gamma$-(1) may be registered in the Position Sensitive Detector (PSD), while $\gamma$-(2) flies directly to the sample where it may be either absorbed, or scattered towards the Backscattering Detector (BSD), where, let us suppose, it is detected. This detection acts as a trigger for the PSD to record the "Event in coincidence" depicted in green on Fig. 1. The PSD records the position of $\gamma$-1) in a matrix, building a bi-dimensional hit pattern whose characteristics depend on the object capabilities to both, scatter and absorb $\gamma$-radiation. For a given sample, the number of coincident backscattering events, i.e. the statistical significance, depends on several parameters like source activity, measuring time, detectors efficiency and geometrical properties. The statistical significance defines the image quality.

\section{Results}

The purpose of the entire technique is to achieve the direct observation of local differences of the sample under study in the field-of-view of the instrument. These local differences are related 


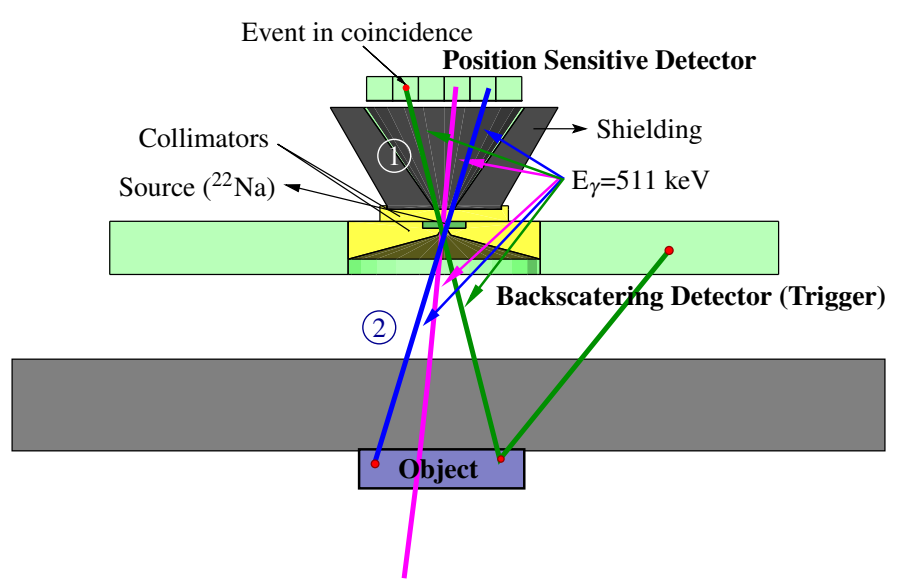

Figure 1: Principle of the backscattering imaging method; see text for details.

to what the interaction $\gamma$-matter can describe: a non uniform mass density or differences in average atomic number. The sensibility of the apparatus is determined by its capability to identify these local differences. In this work, two very simple methods of standard imaging treatment, referencesubtraction and smoothing, were implemented with a clear improvement on the image quality. The first consists of the subtraction of a reference from every image, described in Ref. [2]. Smoothing is, in this work, a very simple mathematical procedure: the counts in each individual pixel are re-calculated using a two-dimensional Gaussian function centered at the interesting pixel of the original image. The number of pixels involved in the average calculation is defined by the standard deviation, $\sigma$, which has to be defined according to some criteria. The weight of each pixel in the average calculation is defined by the value of the Gaussian function at the center of the given pixel. In order to reduce noise as much as possible the optimal $\sigma$ value is defined by the largest value such that the size of the object on the image agrees with the real size and the edge resolution is not compromised. Testing with experimental images taken in very different situations it was determined that $\sigma$ (pixel $)=1.3$ is the optimal value.

All images shown henceforth are the result of the two previous procedures, subtraction and smoothing.

\subsection{Study of materials wear out}

In order to study a non-uniform metallic plate, a possible experimental arrangement is one in which the interest of study focuses in two side-by-side metallic plates placed with the contact line at the center of the field-of-view of the camera. Figure 2 shows a sketch of the setup. The aim of this experiment is to evaluate the capabilities of the camera to study a different mass distribution produced by two objects of the same material but different thicknesses. A first less thorough analysis was already presented in Ref [2].

The Compton Camera was numerically simulated using the Geant4 [4] toolkit. The complete simulated setup includes: BSD, PSD, BSD photomultiplier tubes, aluminum cases of both BSD and PSD, ${ }^{22} \mathrm{Na}$ source, ${ }^{22} \mathrm{Na}$ source holder, printed circuit boards (PCB) and aluminum supports (see Ref. [2] for more details). The BSD was simulated as a monolithic piece of CsI(Na). For the present 

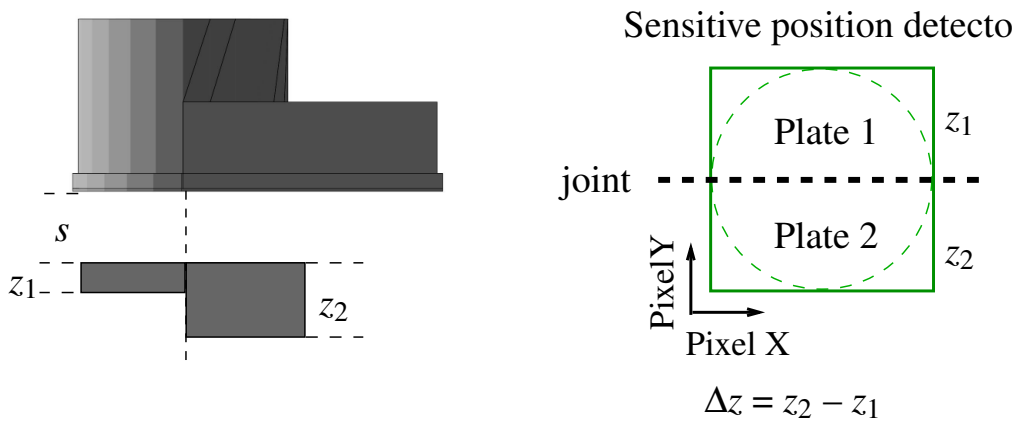

Figure 2: Right: Setup used to test the capabilities of the camera to identify wears in metallic surfaces. Left: Two plates in the field-of-view of the camera.

analysis the PSD was simulated as an array of $64 \times 64$ individual and independent $\mathrm{CsI}(\mathrm{Tl})$ detectors, therefore, in each event the output of the simulated PSD is the pixel where the energy deposition occurs. In order to reproduce photonic transport phenomena each position is recalculated using a bi-dimensional Lorentzian distribution with 5.9 pixels width, centered at the pixel where the energy deposition occurs; the new position is restricted to no more than 7 pixels, in each direction, from the original position. This restriction is equivalent to define a lower energy threshold in the experimental case. Experimental images were obtained for 15 minutes of acquisition time using a ${ }^{22} \mathrm{Na}$ source with $240 \mathrm{kBq}$. The number of events to generate each simulated image was chosen to produce the same number of entries than in the experimental images, i.e. the total number of counts in the matrix is the same in both cases.

Experimental and simulated images are displayed in Figure 3. One plate has fixed thickness $\left(z_{1}=2 \mathrm{~mm}\right.$, Fig. 2$)$. When both plates have the same thickness value, the image represents a rather uniform distribution of counts per pixel. In this case, the interest focuses in thickness difference $(\Delta z)$, for which reason the reference image is defined by both plates with the same thickness $\left(z_{1}=\right.$ $z_{2}=2 \mathrm{~mm}$ ). When the thickness difference is only $2 \mathrm{~mm}$, it is already possible to distinguish the thicker plate, in both experimental and simulated case. The definition of a border line between the plates, that is, the contrast, increases with increasing thickness difference. We can conclude that in this experimental case the contrast (different scattering capacity) is originated in thickness difference.

One can see in Figure 3 that for each $\Delta z$ there is optically no apparent difference between the experimental and its corresponding simulated image. Two features are interesting to be numerically characterized: Edge definition and global similarity between experimental and simulated images. We are going to analyze the images produced for $\Delta z=6 \mathrm{~mm}$ (rightmost panels in Fig. 3). Figure 4 displays the projection of each image on its Y-axis where we can see that the border (limit between the two plates) is defined by 10 or 11 pixels in the simulated image whereas in the experimental one it takes between 11 and 13 pixels. We can see that the new method to simulate the PSD (independent pixels) reproduces the edge resolution and the contrast in a better way in comparison to the results reported in Ref [2], where the simulated images had a rather unrealistic resolution compared to the experimental ones.

Regarding the global similarity or disimilarity we have chosen to calculate the so called 


\section{Experiment}

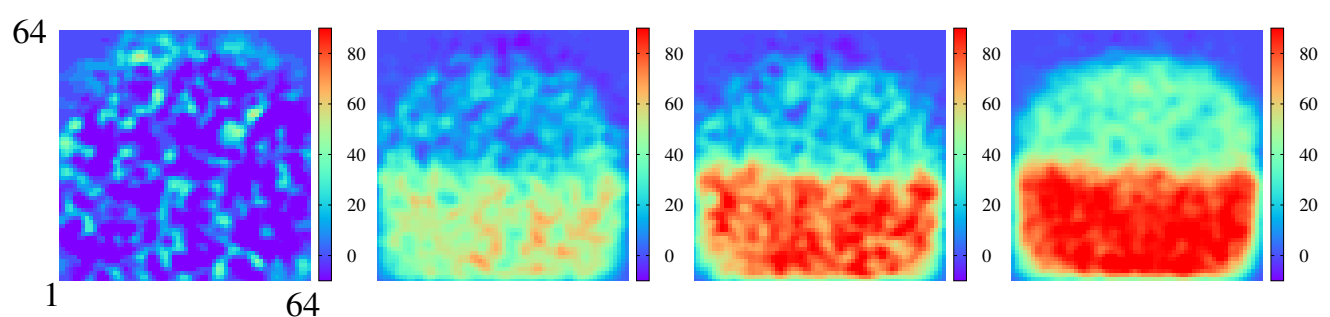

Simulation

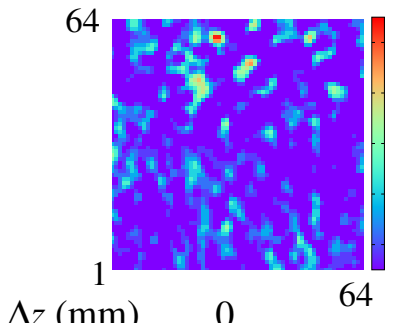

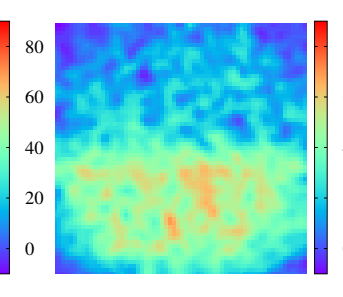

2

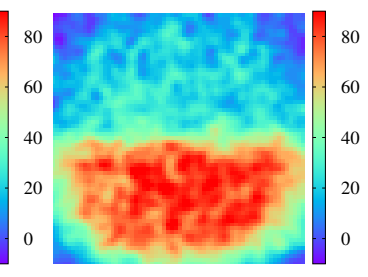

4

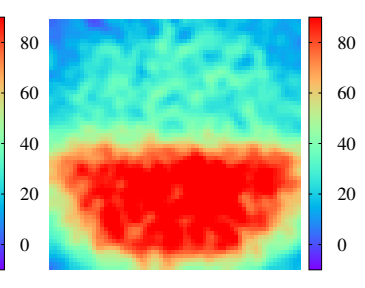

6

Figure 3: Experimental and simulated images of two side-by-side iron plates with different thicknesses placed in front of the camera's field of view. One plate has fixed thickness $z_{1}=2 \mathrm{~mm}$. For this case, the reference image is obtained when both plates have the same thickness. Stand-off distance was $s=3.5 \mathrm{~cm}$.

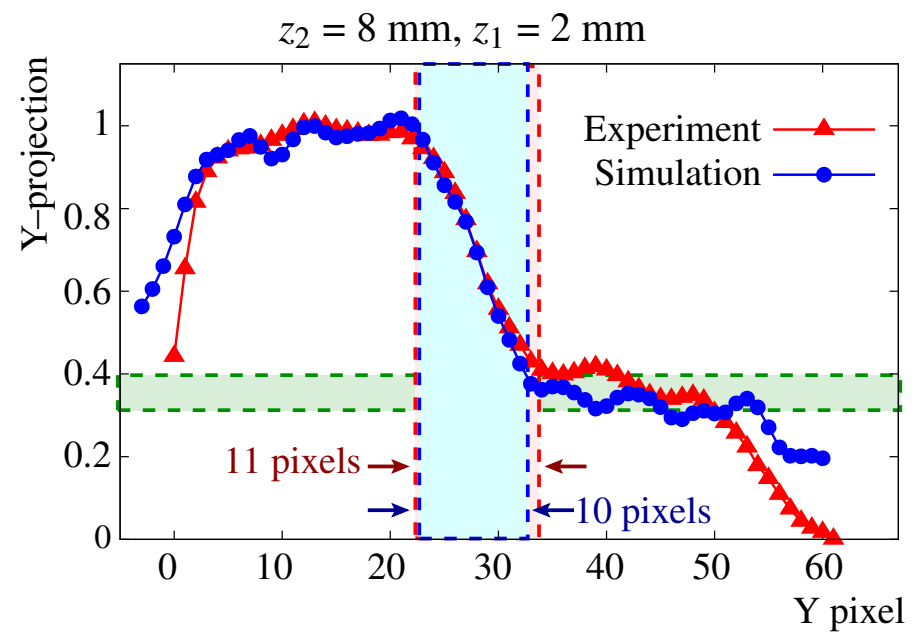

Figure 4: Projection onto the $Y$ axis for experimental and simulated data, when the difference in thickness is $6 \mathrm{~mm}$, normalized to mean counts per pixel in the region defined by the thicker plate.

$\gamma$-index [5]. Figure 5 shows again the experimental and simulated images for $\Delta z=6 \mathrm{~mm}$ together with a $\gamma$-index map to better realize that also this index ascertain the very good agreement between experiment and simulation: The number of counts per pixel in the images meet the acceptance criteria $(\gamma<1)$ in the inner region. The more external region presents understandable differences (brilliant zones in the $\gamma$-index map) because of low statistics in the border and an already identified non-alignment between PSD crystal and the collimation system in the experimental setup. It 
may be of interest that the $\gamma$-index was calculated defining the intensity-difference criterion at 12 counts per pixel (10\% of the maximum intensity) and the distance-to-agreement (DTA) criterion at 5 pixels (half of the edge-resolution).

$$
z_{2}=8 \mathrm{~mm}, z_{1}=2 \mathrm{~mm}
$$

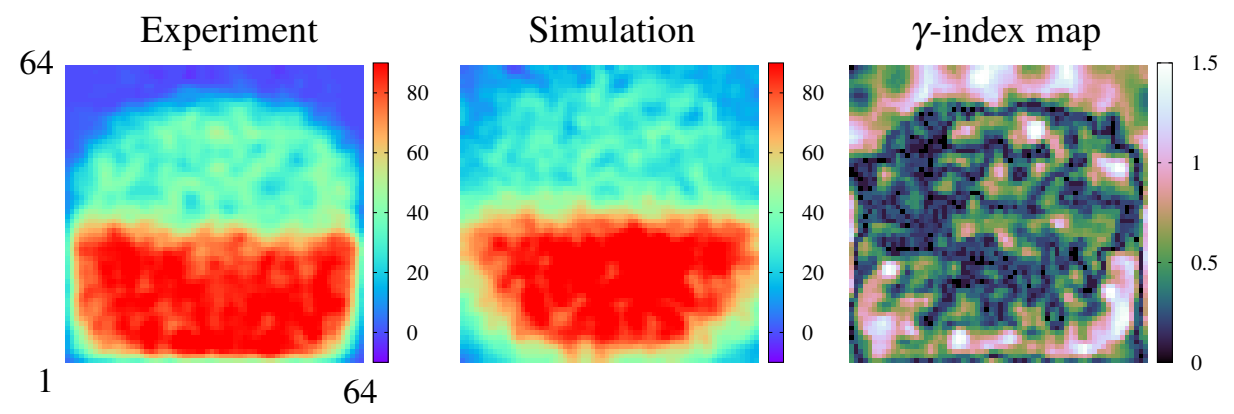

Figure 5: Experimental and simulated images of two side-by-side iron plates when $\Delta z=6 \mathrm{~mm}$. Right: $\gamma$-index map of the simulated image respect to measured ones with DTA $=5$ pixels and the intensitydifference criterion at 12 counts per pixel (10\% of the maximum intensity).

\subsection{Thickness measurement}

In a different situation in which the possible matter defect is larger than the field-of-view of the camera, the interest focuses in the thickness of the sample. In this case, a metallic plate with variable thickness is placed in front to the camera covering the full field-of-view. Figure 6 shows a sketch of the experimental setup and a typical image obtained using this configuration for an aluminum plate $11 \mathrm{~cm}$ thickness.
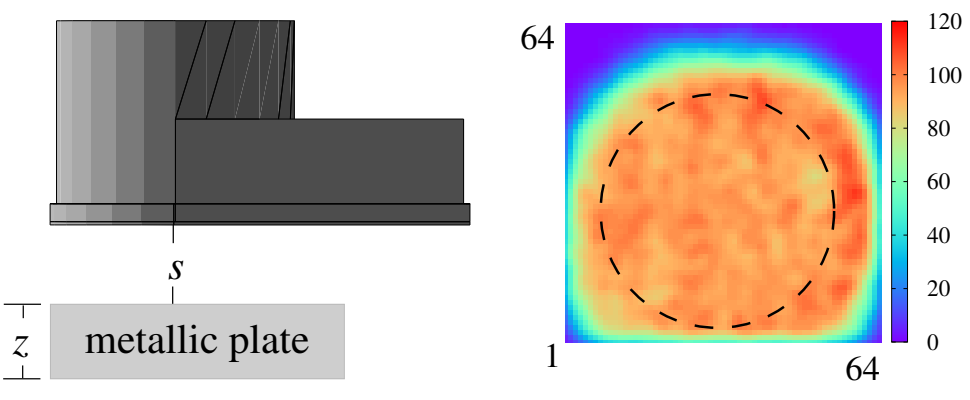

Figure 6: Left: Metallic plate, with variable thickness $(z)$, covering the full field-of-view of the camera $(s$ $=1 \mathrm{~cm}, 2 \mathrm{~cm}$ for an aluminum and iron plate, respectively). Right: Image of an aluminum plate $11 \mathrm{~cm}$ thick. The black dashed line defines the number of pixels taken into account in the average calculation.

The reference image is taken without plate in front of the camera for 8 minutes of acquisition time. The experimental images were obtained by two materials: iron and aluminum.

Taking into account that the full field-of-view is covered completely by the metallic plate, a uniform distribution in the image is obtained. In this case, the intensity of the image increases with the thickness of the plates.

Figure 7 displays the average counts per pixel $(\overline{\mathrm{C}})$ for iron and aluminum plates as a function of the thickness of the plate. Because the border of the image contains no useful information, the number of pixels taken into account in the average calculation is defined by the pixels inside the central region defined by a circumference with 24 pixels radius (black dashed line in Fig. 6). 

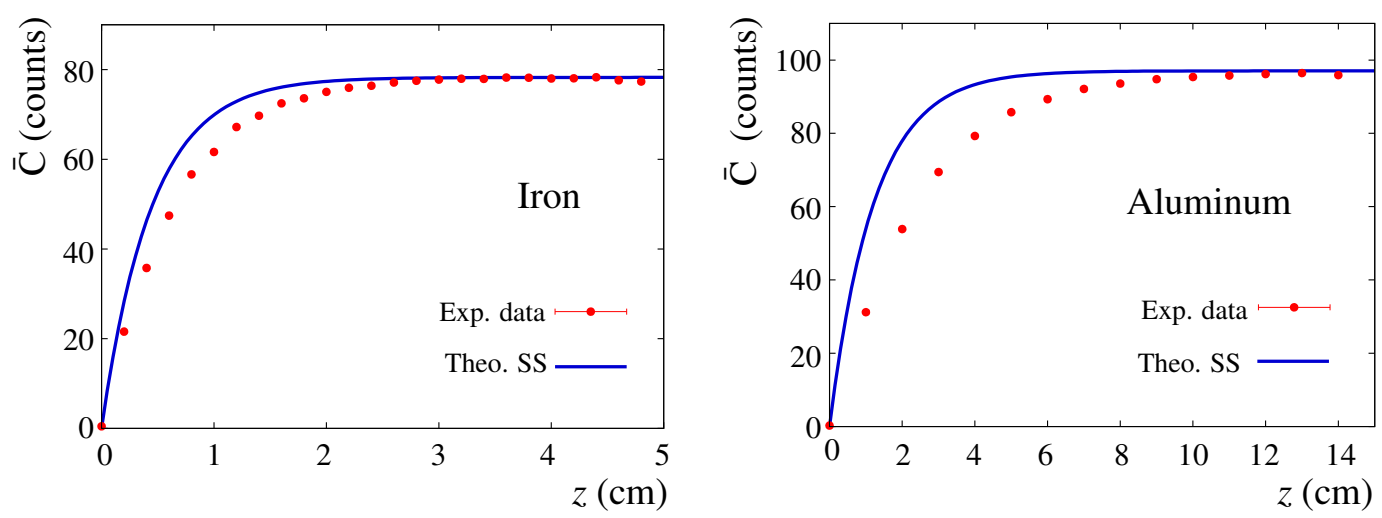

Figure 7: Average of counts per pixel $(\overline{\mathrm{C}})$ as a function of plate thickness for aluminum and iron plates.

Experimental uncertainties are smaller than the point size in Figure 7. The average counts per pixel reaches a saturation value at certain saturation thickness for both samples, similar to the case studied in Ref. [6], around $3 \mathrm{~cm}$ and $10 \mathrm{~cm}$ for iron and aluminum respectively. The saturation depth is, for samples of iron and aluminum, approximately two times the mean free path of $\gamma$ rays with an energy of $E_{\gamma}=511 \mathrm{keV}$ in each material $\left(\lambda_{511 \mathrm{keV}}^{\mathrm{Fe}}=1.52 \mathrm{~cm}\right.$ and $\left.\lambda_{511 \mathrm{keV}}^{\mathrm{Al}}=4.43 \mathrm{~cm}\right)$. The saturation depth depends on the sample composition and defines the experimental limitation of the technique. After a calibration procedure, below the saturation depth the method should be able to measure thickness with certain precision. This precision has to be determined experimentally since it will heavily depend on experimental values such as source activity, acquisition time, geometrical parameters and detector properties.

Ref. [6] presents a theoretical method to obtain the single scattered intensity. It is interesting to compare the experimental results obtained for the Camera with those of the theory because it will tell us about the contribution of the multiple scattered $\gamma$-rays. Figure 7 shows that comparison for the iron and aluminum case. The saturation depth theoretically predicted is approximately one mean free path for $511 \mathrm{keV} \gamma$-rays in each material. This value is half the experimental finding. It is obvious that further improvement in the theory is necessary including multiple scattering.

\section{Perspectives}

The results presented here suggest the possibility of the use of the Compton Camera in several industrial applications. One class of applications has to do with the sheer photographic capacity of the Camera. Further improvements are needed though, for example in the size resolution and the edge definition. On the other hand, besides the imaging capabilities, the total intensity measured may be used towards the purpose of measuring thicknesses of metallic samples.

\section{Acknowledgments}

Parts of this work were performed under research projects DIB 13440 and Colciencias 110152128824. 


\section{References}

[1] Anders Ahnesjö, Collapsed cone convolution of radiant energy for photon dose calculation in heterogeneous media, Med. Phys., 16, 577 (1989).

[2] D. Flechas, L.G. Sarmiento and F. Cristancho, and E. Fajardo, The simulation of an imaging gamma-ray Compton backscattering device using Geant4, Proceedings of the 2013 International Conference on Applications of Nuclear Techniques (CRETE13), Int. J. Mod. Phys. Conf. Ser., 27, 1460152 (2014).

[3] J. Gerl, F. Ameil, I. Kojouharov, and A. Surowiec, High-resolution gamma backscatter imaging for technical applications, Nucl. Inst. Meth. Phys. Res. A, 525, 328-331 (2004).

[4] S. Agostinelli, J. Allison, K. Amako, J. Apostolakis, H. Araujo, P. Arce, M. Asai, D. Axen, S. Banerjee, G. Barrand, F. Behner, L. Bellagamba, J. Boudreau, L. Broglia, A. Brunengo, H. Burkhardt, S. Chauvie, J. Chuma, R. Chytracek, G. Cooperman, G. Cosmo, P. Degtyarenko, A. Dell'Acqua, G. Depaola, D. Dietrich, R. Enami, A. Feliciello, C. Ferguson, H. Fesefeldt, G. Folger, F. Foppiano, A. Forti, S. Garelli, S. Giani, R. Giannitrapani, D. Gibin, J.J. Gómez Cadenas, I. González, G. Gracia Abril, G. Greeniaus, W. Greiner, V. Grichine, A. Grossheim, S. Guatelli, P. Gumplinger, R. Hamatsu, K. Hashimoto, H. Hasui, A. Heikkinen and A. Howard, Geant4 - A Simulation Toolkit, Nucl. Inst. Meth. Phys. Res. A, 506, 250-303 (2003).

[5] D.A. Low, W.B. Harms, S. Mutic and J.A. Purdy, A technique for the quantitative evaluation of dose distributions, Med. Phys, 25, 656-661 (1998).

[6] D. Flechas, L.G. Sarmiento, N. González, J. Gómez-Muñoz, C. Garzón, E. Fajardo, and F. Cristancho The application possibilities of the gamma-ray Compton backscattering technique, AIP Conf. Proc., 1529, 40-44 (2013). 